
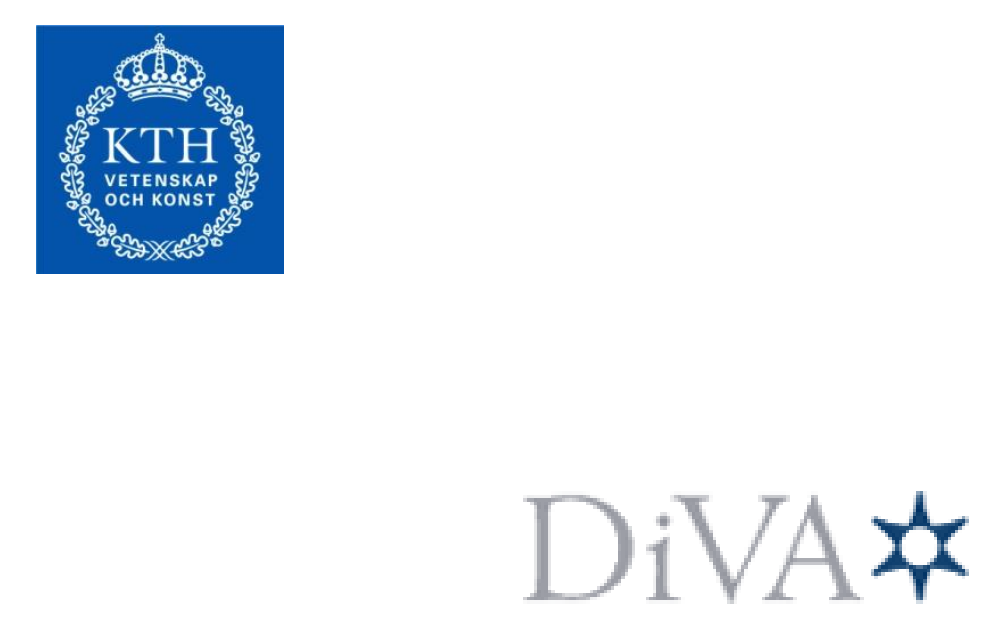

http://kth.diva-portal.org

This is an author produced version of a paper published in 14th International Conference on Transparent Optical Networks (ICTON), 2012.

This paper has been peer-reviewed but does not include the final publisher proofcorrections or proceedings pagination.

(C) 2012 IEEE. Personal use of this material is permitted. Permission from IEEE must be obtained for all other uses, in any current or future media, including reprinting/republishing this material for advertising or promotional purposes, creating new collective works, for resale or redistribution to servers or lists, or reuse of any copyrighted component of this work in other works.

Citation for the published paper:

Aysegul Yayimli and Cicek Cavdar.

Energy-aware virtual topology reconfiguration under dynamic traffic

in 14th International Conference on Transparent Optical Networks (ICTON), 2012.

Access to the published version may require subscription.

Published with permission from: IEEE 


\title{
Energy-Aware Virtual Topology Reconfiguration Under Dynamic Traffic
}

\author{
Aysegul Yayimli, Cicek Cavdar*, Member, IEEE \\ Istanbul Technical University, Maslak, 34469 Istanbul, Turkey \\ * Royal Institute of Technology, Isafjordsgatan 22, 16440 Kista, Sweden \\ Tel: (90212) 2856782,e-mail: gencata@itu.edu.tr, *cavdar@kth.se
}

\begin{abstract}
In an IP over WDM network architecture, IP traffic is carried over a virtual topology (VT), composed of optical transparent channels called lightpaths. We propose an energy-efficient dynamic VT adaptation method with sleep mode, which allows changing only one lightpath connectivity at a time by dynamically monitoring the IP packet traffic load on each lightpath. In order to assure the connectivity of future traffic increase a high-load threshold is used. When the packet traffic load on a specific lightpath becomes higher than a threshold, a new lightpath is added to the virtual topology. However this congestion avoidance policy increases the power consumption by activating transponders in the network. Therefore at the same time lightly loaded lightpaths are eliminated in the virtual topology by using a low-load threshold. Energy-efficient virtual topology adaptation is achieved by traffic offloading considering both lightly loaded and heavily loaded links in the network. In this regard, high- and low-load threshold values need to be carefully determined in order to both gain power and ensure load balancing with keeping the stability of virtual connectivity at a reasonable level.

We have analysed the power consumption of the network during 48-hours for different values of low- and high-load thresholds together with the impact of different threshold values on the stability of the virtual topology. We have shown that there is a trade-off between number of changes in the virtual topology and the energy-efficiency. Experimental results demonstrate that a good compromise can be achieved by adjusting the high- and low-load thresholds carefully.
\end{abstract}

Keywords: Energy-efficiency, IP over WDM networks, optical WDM networks, virtual topology reconfiguration, dynamic traffic.

\section{INTRODUCTION}

Internet has become a significant part of our daily life leading to a rapid increase in traffic rates. Power consumption per user is continuously increasing although power consumption per bit is decreasing with the evolution of power efficient devices in telecommunication networks. In order to meet the requirements of this increasing traffic demands, today's optical wavelength division multiplexing (WDM) networks as a backbone transmission system, is designed to be over-dimensioned, with extra switching capacity and excess number of deployed links, taking into account the peak rates and future growth of the traffic, resulting in waste of energy.

In an IP over WDM network architecture, IP traffic is carried over a virtual topology (VT), composed of optical transparent channels called lightpaths. In transparent optical networks, lightpaths are the most power consuming elements due to the electro-optical operations during the add/drop. Generally, the intensity of traffic on lightpaths follows a daily traffic profile, decreases during the early hours of the morning, starts to increase again after 9 am, and peaks during the daytime. In different time zones, the peak traffic intensities occur at different times, relative to a global clock reference. If the lightly loaded lightpaths can be monitored and put into sleep mode by traffic engineering significant energy can be saved. Virtual topology reconfiguration with the support of standby primitives for line cards is presented in [1] and in [2] authors propose a logical topology design approach periodically solving the problem for each traffic matrix independently for different times of the day which requires to solve an NP complete problem and therefore requires complex optimization methods.

In this study, we propose a simple dynamic VT reconfiguration method with sleep mode, which allows to change only one lightpath connectivity at a time by dynamically monitoring the IP packet traffic load on each lightpath. If the load of a specific lightpath is higher than a high-load threshold a new lightpath is added, if the load is lower than a low-load threshold then the lightpath is put into sleep mode. Here it is important to carefully set high- and low-load thresholds in order to save power without too many changes in VT. We show that proposed method achieves significant saving in power consumption over conventional static VT design approach by assuring the connectivity of possible future traffic increase at the same time.

\section{ENERGY-AWARE DYNAMIC VTR (EAR)}

Energy-aware dynamic VTR (EAR) problem can be stated as follows:

Given: the physical topology $\mathrm{G}(\mathrm{N}, \mathrm{E})$ of $\mathrm{N}$ nodes and a set of edges $\mathrm{E}$, the number of wavelengths $\mathrm{W}$, the lightpath capacity, the number of transponders at the nodes, energy consumption values of each network equipment presented in the following section and the IP layer packet traffic at different times of the day. 
Find: At a given time of the day, which lightpath to activate to provide connectivity in high traffic load and which lightpath to put into sleep mode to save energy in low load.

Solution: In order not to disturb the traffic in the IP layer, algorithm changes only one lightpath at a time in the topology. Periodically, e.g., every 5 minutes, load changes are monitored on the existing lightpaths due to the IP packet traffic fluctuation. Whenever a lightpath load hits the high threshold, a new lightpath is activated. To reduce the bottleneck load, the new lightpath is set up between the nodes whose traffic contributes the most to the load of the maximally loaded lightpath. Whenever a lightpath has a lower load than the low threshold, this lightpath is torn down after rerouting its packet traffic. If more than one lightpaths are below the threshold, the one with the lowest load is deleted. This way, the algorithm tries to keep all lightpath loads between predetermined threshold values. This provides load balancing, and in time the system naturally selects the lightpaths that can best accommodate the current traffic loads which fluctuate continually.

\subsection{Power Model}

We assume an IP over WDM network composed of the virtual topology and the underlying physical topology where the node architecture is similar to [8]. Each node is composed of an optical cross connect and a packet router, e.g., an IP router where short reach interfaces are assumed between the router and the optical switch. Each lightpath corresponds to a line card and a transponder which can be put into sleep mode. In each IP router, line cards are connected to transponders which perform EO and OE conversions. Cisco CRS-1 router is taken into account in the model, and "Single-Port STM-256c POS Interface Module" is selected with the line card: "Modular Services Card" and transponder: "Tellabs 7100 Optical Transport System 40 Gigabit Transponder Module". Total power consumption of these devices can be calculated as 667W [2] and represented in the following formula as $\varepsilon^{e}$. Therefore, total energy consumption in an IP-over-WDM network can be analyzed by:

$$
P O W=\sum_{\forall(m, n) \in E} a_{m n} u_{m n}+\sum_{\forall i \in N} \emptyset_{i} n_{i}+\rho \sum_{\forall(i, j) \in D} \varepsilon^{e} \lambda_{i j}+(1-\rho) \sum_{\forall(i, j) \in D} \varepsilon^{e} l_{i j}+\sum_{\forall(m, n) \in E, m \neq s, d} \varepsilon^{s} p_{m n}
$$

Here, $a_{m n}$ is the energy consumption of in-line amplifiers on physical link $(\mathrm{m}, \mathrm{n}) . u_{m n}$ is a binary variable equal to 1 if a wavelength on link $(m, n)$ is used by a lightpath. $\emptyset_{i}$ is traffic independent (idle power) energy consumption at node $\mathrm{i}$ by the active devices and $n_{i}$ is a binary variable equal to 1 if node $i \in N$ is used by any lightpath. $\rho$ is the idle (traffic independent) percentage of $\varepsilon^{e}$, where $\varepsilon^{e}$ is energy consumption per lightpath. When $\lambda_{i j}$ is 1 there is a lightpath between nodes $\mathrm{i}$ and $\mathrm{j} .(1-\rho)$ is the traffic dependent percentage of $\varepsilon^{e} . l_{i j}$ is the traffic load on lightpath (i,j). $\varepsilon^{s}$ is the energy consumption per wavelength for switching devices at a node such as a MEMS optical switch. $p_{m n}$ is the number of wavelengths in use on physical links $(\mathrm{m}, \mathrm{n})$.

The first two terms in equation (1) define the idle power for physical links and nodes, respectively. The third and fourth terms correspond to idle and traffic dependent power consumption per lightpath at the starting and terminating nodes respectively in IP layer, i.e., EO and OE conversions, electronic processing at the IP port, and the fifth term is the power consumption at the pass-through nodes.

Power consumption parameters are set according to [2], [4] and [5] as follows: Electronic control power consumption at each node is $\emptyset_{n}=150 \mathrm{~W} \forall \mathrm{n} \in \mathrm{N}$. Electronic processing per IP port, $\varepsilon^{e}=667 \mathrm{~W}$ for $40 \mathrm{Gbps}$ channel capacity and the idle percentage of this power, $\rho=0.9$. 3D MEMS switching per wavelength consumes a total $\varepsilon^{s}=0.107 \mathrm{~W}$ of power. Power consumed by in-line amplifiers is calculated by the formula $a_{m n}=\varepsilon^{a} . A_{m n}$ where $\varepsilon^{a}=9 \mathrm{~W}$ and $A_{m n}=\left\lfloor d_{m n} / 80 \mathrm{~km}+2\right\rfloor$ including pre and post amplifiers. $d_{m n}$ is the length of physical link $(\mathrm{m}, \mathrm{n})$ in $\mathrm{km}$.

\section{NUMERICAL EXAMPLES}

To illustrate the performance of EAR method, the effect of high and low threshold values over the power consumption is analyzed. We compared the results with the conventional VT design approach, Static-VT, where VT is designed considering peak traffic rates. In order to have a fair comparison we used two different StaticVT, one with a high load threshold of $80 \%$ and a low threshold of $20 \%$ and the other one with a high load threshold of $60 \%$ and a low threshold of $10 \%$. We assume a 24-node network [6] with 8 transponders per node, and 16 wavelengths per fiber. The network is assumed to have no wavelength converters. All lightpaths have the same capacity (40 Gbps). The IP traffic is routed over the virtual topology using shortest paths.

The traffic intensities between node pairs is modeled for 24 hours, imitating several real network links as in [3]. While the traffic intensities gradually changes between node pairs, the lightpath loads are observed every 5 minutes. The simulation starts with a predesigned virtual topology, and results are reported after a warm up period showing the simulation of a 48 -hour period.

Figure 1 shows the comparison of the power required by the whole network during a period of two days for the EAR algorithm with different high and low threshold values (60-10,60-20, 80-10, and 80-20), and for the case of Static-VT (80-20, 60-10). As shown in the figure, EAR can adjust the power consumption of the network by the help of high and low threshold values. Figure 1 shows that for all threshold sets, EAR outperform StaticVT in power consumption. High threshold values allows more loads to be carried by the existing lightpaths and 
new lightpaths are not necessarily added, therefore, EAR-80-20 has significantly more power gain than EAR-6020. On the other hand, when the high threshold values are smaller, more lightpaths are activated, thus increasing the power consumption. This way the high threshold value can be used to control the energy spent for the networks operations, the frequency of topology updates, and the maximum load carried by lightpaths. Furthermore, when we increase the low threshold value from 10 to 20, EAR gains in power consumption with the cost of number of changes in the topology.

The addition and deletion of lightpaths during the operation of the network are undesirable. However, making no changes at all, and using a simple virtual topology design heuristic to accommodate the current and future traffic can be very inefficient in terms of power consumption. With EAR-80-10 no change is made, however, it still possible to gain a $4.1 \%$ over the static VT $80-20$ and a $22.6 \%$ over the static VT $60-10$. This is mainly due to the adaptation to the traffic during the warm up period in EAR-80-10. EAR-80-20 saves $21.2 \%$

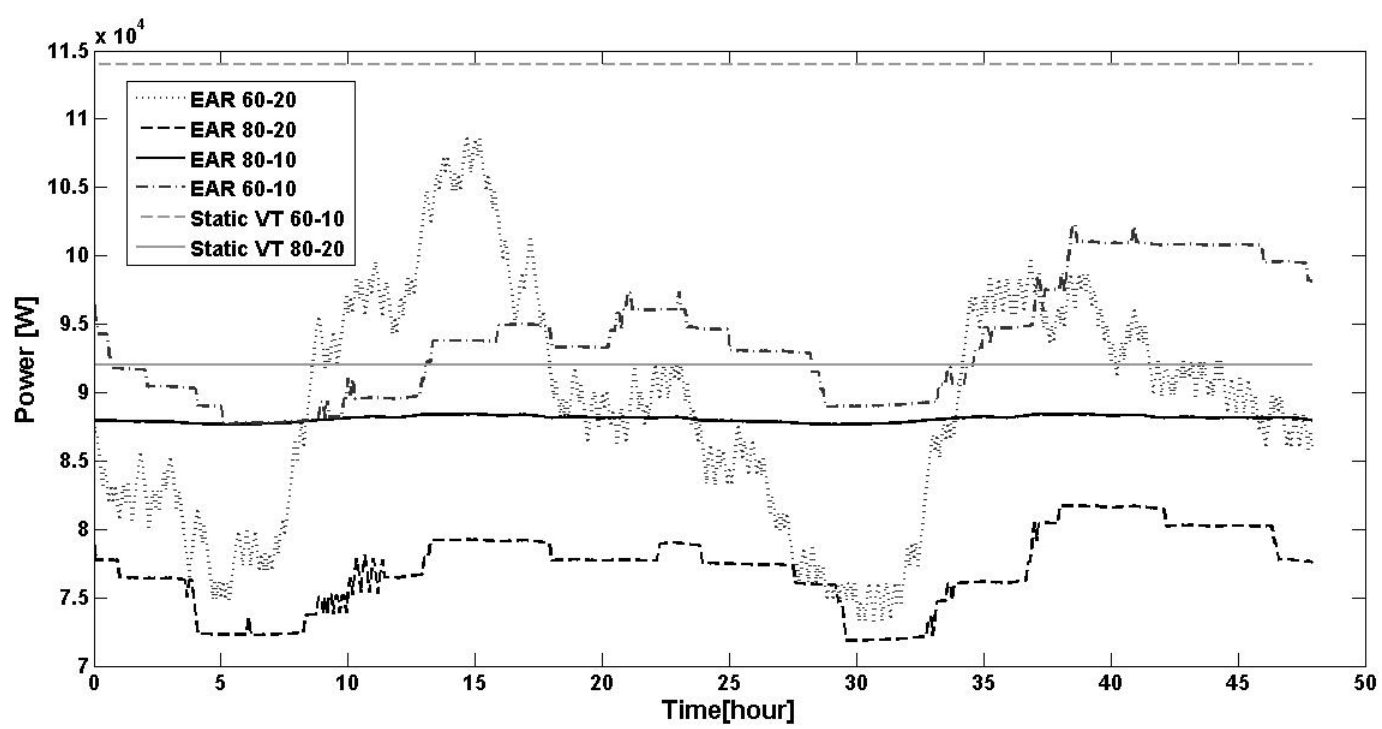

Figure 1. Power required during 48 hours.

power for the low traffic rate, and $11.9 \%$ power for the peak traffic rate compared to Static VT $80-20$, which is the best case of Static VT in terms of energy. This saving comes with a larger number of changes in the virtual topology when the low threshold is increased from 10 to 20. It should be noted that the power gain in the peak rate with EAR is due to the consideration of traffic fluctuation based on time-zones, and nodes located in different time zones. Moreover, EAR-60-10 saves 22.8\% power for the low traffic rate and 9.1\% power for the peak traffic rate compared to Static VT 60-10. Finally, with EAR-60-20, which is the case with more changes, $34.2 \%$ power over the Static VT $60-10$ is saved for the low traffic rate, and $9.0 \%$ power for the peak traffic rate.

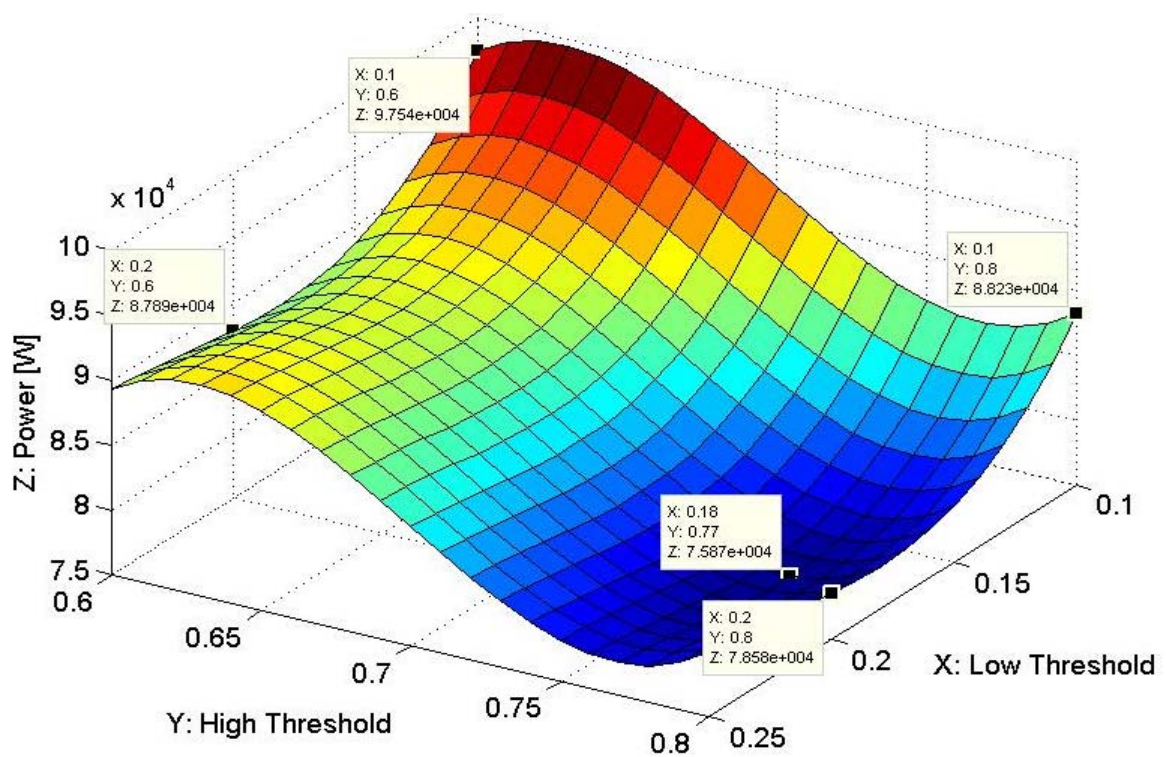

Figure 2.Average power consumption vs. high- and low-load thresholds. 
Figures 2 and 3 show the average power required in total by the whole network in 48-hours vs. different high and low threshold values after a warm up period and we can observe the effect of these thresholds on the power savings. The highest consumption occurs when the thresholds are 60 and 10 percentage and the lowest when the thresholds are 80 and 20 percentage. An interesting observation is that EAR-80-10 and EAR-60-20 have a very similar consumption with different behaviours. EAR-60-20 does a lot of changes in VT, while EAR-80-10 reaches a very stable VT after warming up period (see Fig. 4). It could seem that EAR-80-10 is better because we have the same consumption with less number of changes in the topology, but with EAR-60-20 we are also maintaining load balancing in all lightpaths, which is desirable for the stability of the network. It should be noted that with high values in the high threshold we are gaining energy but with the risk of high loads in lightpaths which could be dangerous if a traffic burst arrives to the network. So, EAR-80-20 saves 12.3\% power compared to EAR-60-20 and EAR-80-10 saves 5.6\% power compared to EAR-60-10. In the other hand, with high values in the low threshold we are gaining energy with the cost of number of changes in the network. Thus, EAR-80-20 saves $12.6 \%$ power compared to EAR-80-10 and EAR-60-20 saves 6.0\% power compared to EAR-60-10. The trade-off depends on the limits you can set in your network.

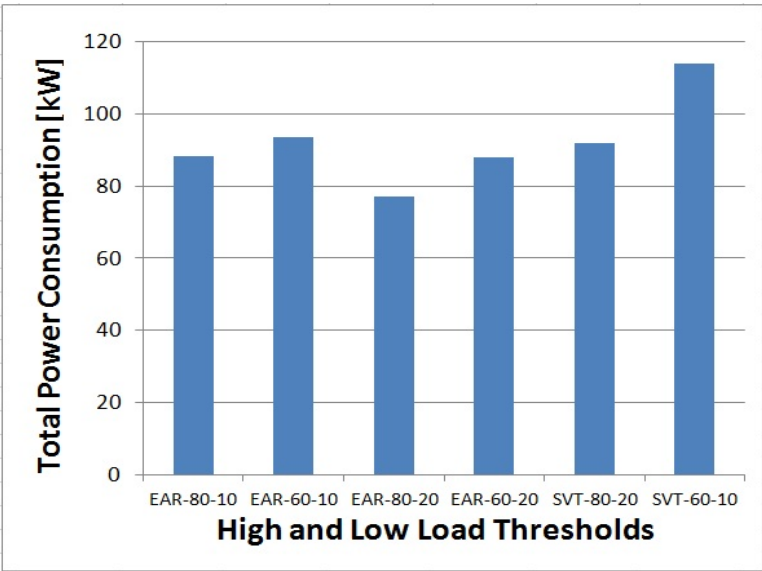

Figure 3. Average power cons. in 48 hours.

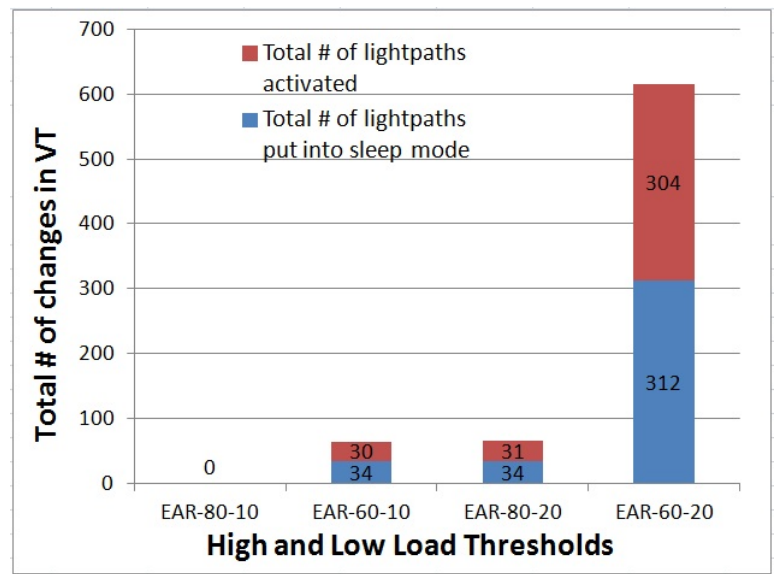

Figure 4. Number of changed lightpaths.

In Fig. 4 we show the total number of lightpaths added and deleted in the virtual topology during 48-hours of simulation. The highest number of changes is made when the thresholds are 60 and 20, since this is the case where the lightpath loads are most severely controlled, in our example.

\section{CONCLUSIONS}

We propose an energy-aware dynamic virtual topology reconfiguration algorithm (EAR) exploiting IP packet traffic fluctuations. We show that EAR continuously reconfigures the virtual topology with a simple effort following the traffic trends with significant savings in power consumption. EAR-80-10 saves $4.1 \%$ over the static VT $80-20$ by putting lightpaths with load of less than $10 \%$ into sleep mode. Further savings can be achieved by adjusting the lower load threshold to 20, which leads to higher number of lightpaths in sleep mode. Using EAR-80-20 up to $21.2 \%$ power savings over the static VT 80-20 could be obtained when the topology is allowed to change with the dynamic traffic.

\section{REFERENCES}

[1] R. Bolla, R. Bruschi, A. Cianfrani, M. Listanti: Enabling backbone networks to sleep, IEEE Network, 25 (2), pp. 26-31, 2011.

[2] E. Bonetto, L. Chiaraviglio, D. Cuda, F. Idzikowski, F. Neri: Exploiting traffic dynamics in power-aware logical topology design, in Proc. ECOC2011.

[3] A. Gencata, B. Mukherjee: Virtual-topology adaptation for WDM mesh networks under dynamic traffic, IEEE/ACM Trans. on Networking, 14, pp. 236-247, 2004.

[4] S. Aleksic: Analysis of power consumption in future high-capacity network nodes, IEEE J. Opt. Commun. Netw., 1, pp. 245-258, 2009.

[5] R.S. Tucker: Are optical networks green?, in Proc. OFC2010, W. on Green Optical Networks.

[6] B. Mukherjee: Optical WDM Networks, Springer-Verlag, 2006.

[7] E. Leonardi, M. Mellia, M. A. Marsan: Algorithms for the logical topology design in WDM all-optical networks, Optical Networks Mag., 1, pp. 35-46, 2000.

[8] R. Hulsermann et al.: Cost modeling and evaluation of capital expenditures in optical multilayer networks, Journal of Optical Networking, 7, pp. 814-833, 2008. 